\title{
A SIMPLE AND EFFICIENT THREE-NODE CURVED BEAM ELEMENT FOR THE OUT-OF-PLANE SHEAR, BENDING AND TORSION BASED ON THE LINKED INTERPOLATION CONCEPT
}

\begin{abstract}
Summary
The paper presents a new three-node curved beam finite element for out-of-plane actions constructed on the expanded linked interpolations used before on a straight beam. The shear strain constraints are immanent in displacement interpolations and the geometry is interpolated compatibly with the used kinematics, so that the element stiffness forming procedure is very simple and can easily be implemented in any programming code.

Different than the standard, an equivalent curved beam kinematics is introduced, which enables the use of global displacement variables instead of mostly used displacement parameters in local coordinates, avoiding the coordinate transformation for nodal degrees of freedom.

Timoshenko beam theory assumptions are taken into account and the element was tested on a set of benchmark problems to demonstrate that the element shows no shear or membrane locking.
\end{abstract}

Key words: $\quad$ curved beam element, out-of-plane actions, Timoshenko beam theory, problem-independent linked interpolation

\section{Introduction}

The problem of modelling curved linear structures with the finite element method has already been widely analysed, and many curved beam elements have been presented in the literature for that purpose. Among them, plane curved elements with out-of-plane actions attracted minor interest, but there are still quite a number of good elements in the curved form capable of capturing bending, torsion and shear strains in curved element formulations. The literature overview points to two different approaches covering the subject.

Typically, a curved beam element is modelled with a constant geometry curvature (constant radius) in the beam plane with three unknown parameters per node (degrees of freedom), defined in the local coordinates, $w_{i}, \theta_{B, i}$ and $\theta_{T, i}$, representing the out-of-plane displacement, the bending section rotation and the twist section rotation around the normal and tangential axes of the curved beam section, respectively. Knowing the geometric curvature radius of the beam, the exact solution of the differential equations gives displacements and stress resultants as trigonometric functions. If the curved element is 
modelled with only two nodes and the unknown displacements are simply linearly interpolated along the beam's axis, these interpolations actually underestimate the geometry of the beam, which it is not linear. Obviously, some locking problems must occur [1]. The solution to that problem lies in increasing the polynomial order for the displacement interpolations, e.g. by introducing more nodes in the element model [2] or by enhancing interpolations with higher order penalty terms depending on the type of locking [3]. Generally, it was concluded in [2] and [3] that the use of the same polynomial order for all displacement field interpolations introduces inconsistency in the strains. On the other hand, the problem of inherent locking can be overcome by introducing a mixed field approach, where, besides the deformations, the strains are also interpolated separately [4], [5]. However, whenever the curvature radius is held constant in the finite element description, a problem of approximating a straight beam exists with the radius becoming infinite and the trigonometric solution getting an indeterminate expression. That situation results in another kind of locking caused by the simple straight geometry of the beam.

Another approach is to approximate the beam geometry as well as beam displacements in the same or similar way, e.g. by the polynomials of the quadratic or higher order. Since the curved form of the beam can be modelled at least by three nodes, the Lagrangean quadratic polynomial is appropriate for all interpolations and the element is isoparameric [1]. The three nodes can easily be formed in the straight line and no locking should be expected out of the beam geometry. Again, some other kind of locking cannot be avoided, such as the "thin limit" locking, where the derived shear strains produce too stiff element models, unless higher order polynomials are chosen for the deflection field rather than for the rotations. Geometry of the curved element [6] is modelled with five nodes as is the deflection field, but the rotations are interpolated with four node parameters (the element middle node is left out). Furthermore, in [7] the locking problem is overcome by a mixed formulation: the shear field is interpolated linearly, the twist rotation is interpolated quadratically and the deflection is cubic, while the bending rotation is not interpolated at all. Both methods avoid locking of any kind and can reproduce the straight beam problem with constant shear exactly in every point.

The standard procedure in the most commercial finite element programs uses the Lagrangean interpolations for all fields (displacement and rotations) on two or three-node beam elements and the reduced integration technique in the stiffness matrix formulation, actually resulting in constant strains for the two-node beam element or linear strains for the three-node beam element.

The linked interpolation technique uses the Lagrangean interpolation for the rotations and one-order higher polynomials for the displacement, with the higher order terms related to the nodal rotations. The linking expression for the displacement is chosen to produce constant strain fields for the two-node element and linear strains for the three-node element. The same result is obtained by applying the reduced integration technique. It would be demonstrated here that the linked interpolation could efficiently be used even for the curved beam forms in the out-of-plane problems, with even better performances than the standard procedure.

\section{Basic equations}

In defining the problem of a curved beam with its geometry described in the $x-y$ plane while subjected to out-of-plane forces, among all three space displacements and three space rotations of the beam cross-section, only the transverse displacement $\mathrm{w}$ and two rotations around the local in-plane axes $\theta_{\eta}$ and $\theta_{\xi}$ are significant. Related to them, only cross-sectional bending moment $M_{B}$, torsional moment -torque $M_{T}$ and transverse shear force $V_{z}$ are active stress resultants, see Fig. 1.

The problem of the curved beam is usually defined in the literature by three sets of equations [1], [4], [7-12]. According to the orientations of the assumed global and local 
coordinates as well as to the assumed orientation of the beam geometry radius, the equations in the cited literature can have different signs. According to the positive orientations from Fig. 1, the following equilibrium equations for the differential segment of the beam, placed in the $\mathrm{x}-\mathrm{y}$ plane and subjected to the out-of-plane actions, are set:

$$
\begin{aligned}
& \sum Z=0: \frac{\mathrm{d} V}{\mathrm{~d} s}=-q_{z}, \\
& \sum M_{\eta}=0: \frac{\mathrm{d} M_{B}}{\mathrm{~d} s}+\frac{M_{T}}{R}-V=0, \\
& \sum M_{\xi}=0: \frac{\mathrm{d} M_{T}}{\mathrm{~d} s}-\frac{M_{B}}{R}=0 .
\end{aligned}
$$

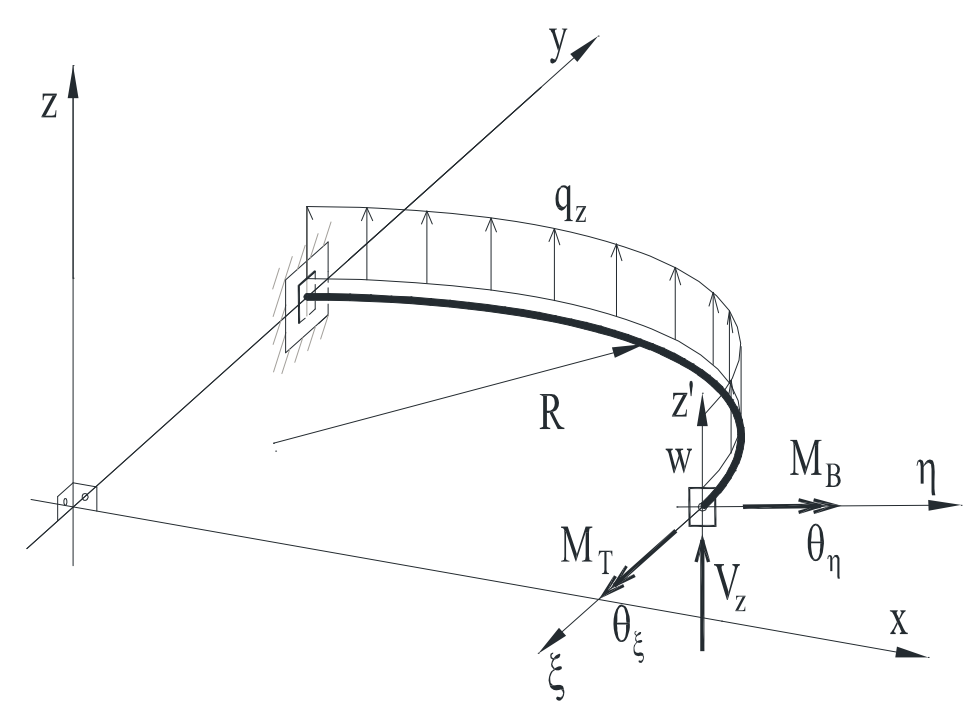

Fig. 1 Curved beam - involved displacements and stress resultants for out-of-plane actions. Positive orientations are depicted.

Then, the kinematic and constitutive equations for the linear analysis are also obtained:

$$
\begin{aligned}
& \gamma_{z}=\frac{\mathrm{d} w}{\mathrm{~d} s}+\theta_{\eta}, \\
& \kappa_{B}=\kappa_{\eta}=\frac{\mathrm{d} \theta_{\eta}}{\mathrm{d} s}+\frac{\theta_{\xi}}{R}, \\
& \kappa_{T}=\kappa_{\xi}=\frac{\mathrm{d} \theta_{\xi}}{\mathrm{d} s}-\frac{\theta_{\eta}}{R}, \\
& \gamma_{z}=\frac{V}{G k A}, \\
& \kappa_{B}=\frac{M_{B}}{E I_{B}}, \\
& \kappa_{T}=\frac{M_{T}}{G I_{T}} .
\end{aligned}
$$

Among these nine equations, the most important are kinematic equations (4)-(6), as the equilibrium in the finite element modelling will be imposed by the total energy minimization procedure, while constitutive relations (7)-(9) are simple transformations of the strains to the 
stress resultants in the linear elasticity material conditions. In kinematic equations, only (5) and (6) are affected by the curved beam geometry. Equation (4) is actually identical to that of the straight beam.
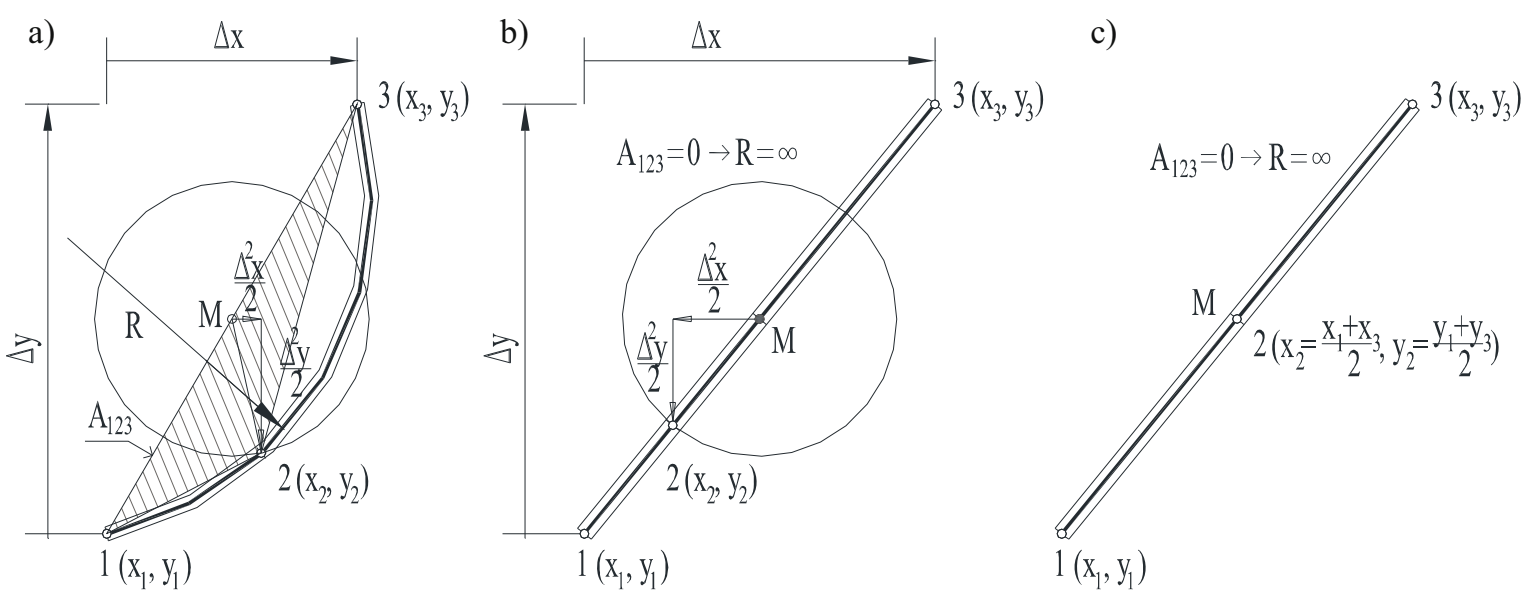

Fig. 2 Possible distortions of three-node beam configuration: a) general quadratic curved beam,

b) straight but quadratic beam, c) straight linear beam

Then, the position on the beam axis is determined with the Lagrangean interpolation, defined on the bi-unit domain $[-1,1]$ by a position parameter $\xi$ :

$$
\begin{aligned}
& x=(-\xi)\left(\frac{1-\xi}{2}\right) \cdot x_{1}+4\left(\frac{1-\xi}{2}\right)\left(\frac{1+\xi}{2}\right) \cdot x_{2}+\xi\left(\frac{1+\xi}{2}\right) \cdot x_{3}=x_{2}+\frac{\Delta x}{2} \xi+\frac{\Delta^{2} x}{2} \xi^{2}, \\
& y=(-\xi)\left(\frac{1-\xi}{2}\right) \cdot y_{1}+4\left(\frac{1-\xi}{2}\right)\left(\frac{1+\xi}{2}\right) \cdot y_{2}+\xi\left(\frac{1+\xi}{2}\right) \cdot y_{3}=y_{2}+\frac{\Delta x}{2} \xi+\frac{\Delta^{2} y}{2} \xi^{2},
\end{aligned}
$$

with $\left(x_{1}, y_{1}\right),\left(x_{2}, y_{2}\right),\left(x_{3}, y_{3}\right)$ as co-ordinates of the three arbitrary chosen nodes of which the first and the third node are taken to be at the beginning and at the end of the beam and the second one is an arbitrary axis position. The abbreviations $\Delta x$ and $\Delta y$ stand for the total segment projection differences $\left(\Delta x=x_{3}-x_{1}, \Delta y=y_{3}-y_{1}\right)$, and the quadratic geometric differences are expressed as $\Delta^{2} x=x_{1}-2 x_{2}+x_{3}$ and $\Delta^{2} y=y_{1}-2 y_{2}+y_{3}$.

The beam axis curvature present in (2)-(3) and (5)-(6) is a directional quantity, which can be expressed in terms of position parameter $\xi$ as

$$
\frac{1}{R}=\frac{\mathrm{d}^{2} y}{\mathrm{~d} s^{2}} \frac{\mathrm{d} x}{\mathrm{~d} s}-\frac{\mathrm{d}^{2} x}{\mathrm{~d} s^{2}} \frac{\mathrm{d} y}{\mathrm{~d} s}=\frac{\frac{\mathrm{d}^{2} y}{\mathrm{~d} \xi^{2}} \frac{\mathrm{d} x}{\mathrm{~d} \xi}-\frac{\mathrm{d}^{2} x}{\mathrm{~d} \xi^{2}} \frac{\mathrm{d} y}{\mathrm{~d} \xi}}{\left(\frac{\mathrm{d} s}{\mathrm{~d} \xi}\right)^{3}}
$$

and can be formulated for the parabolic beam geometry as

$$
\frac{1}{R}=\frac{\Delta^{2} y \cdot\left(\frac{\Delta x}{2}+\Delta^{2} x \cdot \xi\right)-\Delta^{2} x \cdot\left(\frac{\Delta y}{2}+\Delta^{2} y \cdot \xi\right)}{\left(\frac{\mathrm{d} s}{\mathrm{~d} \xi}\right)^{3}}=\frac{\Delta^{2} y \cdot \Delta x-\Delta^{2} x \cdot \Delta y}{2\left(\frac{\mathrm{d} s}{\mathrm{~d} \xi}\right)^{3}}=\frac{2 A_{123}}{\left(\frac{\mathrm{d} s}{\mathrm{~d} \xi}\right)^{3}},
$$

with $\quad \frac{\mathrm{d} s}{\mathrm{~d} \xi}=\sqrt{\left(\frac{\Delta x}{2}+\Delta^{2} x \cdot \xi\right)^{2}+\left(\frac{\Delta y}{2}+\Delta^{2} y \cdot \xi\right)^{2}}=J$. 
$J$ being the Jacobian of the local to global coordinate mapping, and where $A_{\Delta 123}$ is the area of the triangle formed by the three beam element nodes. The curvature of the beam is a rational function of variable $\xi$, with a constant numerator and a variable part in denominator $J^{3}$.

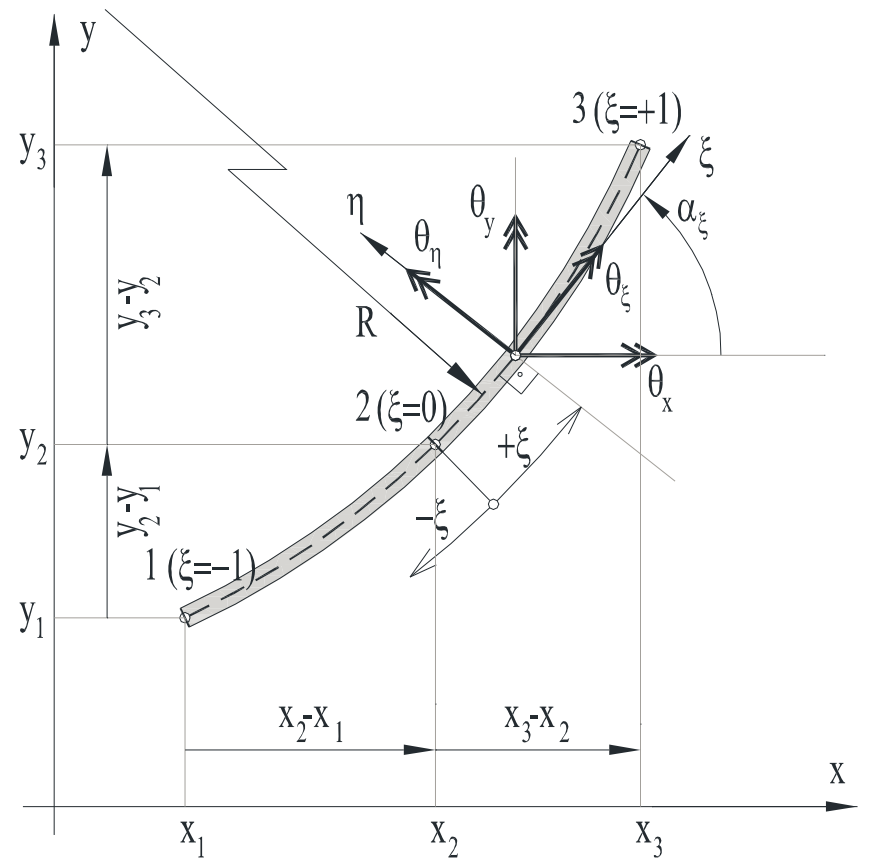

Fig. 3 Geometry of the curved beam problem and positive orientations of involved variables

\section{Curved beam distortions}

With an infinite geometry curvature radius, the beam is just a standard straight beam with two nodes connected in the shortest way. Further, if intermediate nodal point " 2 " is selected to be at the middle point $\mathrm{M}$ of the straight line connecting end node points " 1 " with "3", then:

$$
x_{2}=\frac{x_{1}+x_{3}}{2} \text { and } y_{2}=\frac{y_{1}+y_{3}}{2}
$$

and the quadratic differences will vanish $\left(\Delta^{2} x=0\right.$ and $\left.\Delta^{2} y=0\right)$, meaning that the straight beam element is linear in geometry (example c) in Fig. 2). The beam can still be straight even when (15) is not satisfied (example b) in Fig. 2), but this beam should be considered as quadratic since $\Delta^{2} x \neq 0$ and $\Delta^{2} y \neq 0$, like any other curved form beam of example a) in Fig. 2.

The geometry curvature (12) and its beam geometry radius are signed values. Nodes 1 , 2 , and 3 define a parabolic curved beam geometry in (10), (11) and (13), while the pertaining curvature is positive if the nodes in a "triangle 1-2-3" are positively oriented (Fig. 2).

\section{Alternative kinematics}

Since the chosen kinematic equations are the most sensitive issue in modelling curved beams subjected to out-of-plane actions, let us examine how they can be justified. Firstly, we shall consider the standard kinematics ("kinematics 1") on the beam segment of differential length $\mathrm{d} s$. There are two independent rotations around the local main axis $\theta_{\xi}$ and $\theta_{\eta}$ of the initial cross-section and the increased values of these rotations at the other segment end. Due 
to the curved form of the differential segment, the total change of these rotations around the local axis of the end cross-section is equal to the total curvature changes in the $\xi$ and $\eta$ directions (Fig. 4), and they are torsional deformation curvature $\kappa_{\xi} \cdot \mathrm{d} s$ and bending deformation curvature $\kappa_{\eta} \cdot \mathrm{d} s$.

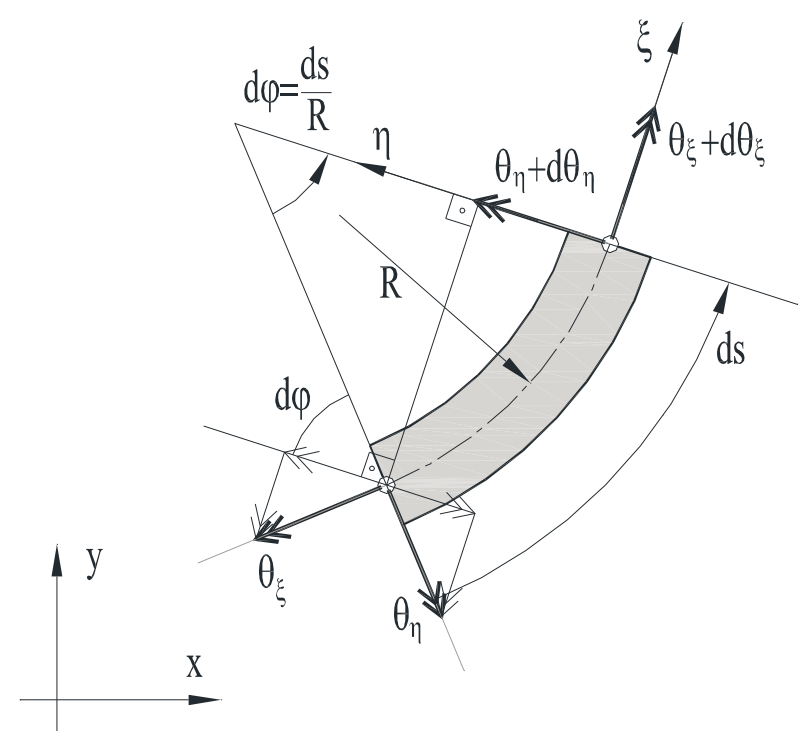

Fig. 4 Kinematics 1

In direction $\eta$ it is: $\quad \kappa_{\eta} \cdot \mathrm{d} s=\theta_{\eta}+\mathrm{d} \theta_{\eta}-\theta_{\eta} \cos \mathrm{d} \varphi+\theta_{\xi} \sin \mathrm{d} \varphi$, and in direction $\xi$ it is: $\kappa_{\xi} \cdot \mathrm{d} s=\theta_{\xi}+\mathrm{d} \theta_{\xi}-\theta_{\xi} \cos \mathrm{d} \varphi-\theta_{\eta} \sin \mathrm{d} \varphi$.

From $\cos \mathrm{d} \varphi \cong 1$ and $\sin \mathrm{d} \varphi \cong \mathrm{d} \varphi=\frac{\mathrm{d} s}{R},(5)$ and (6) are obtained.

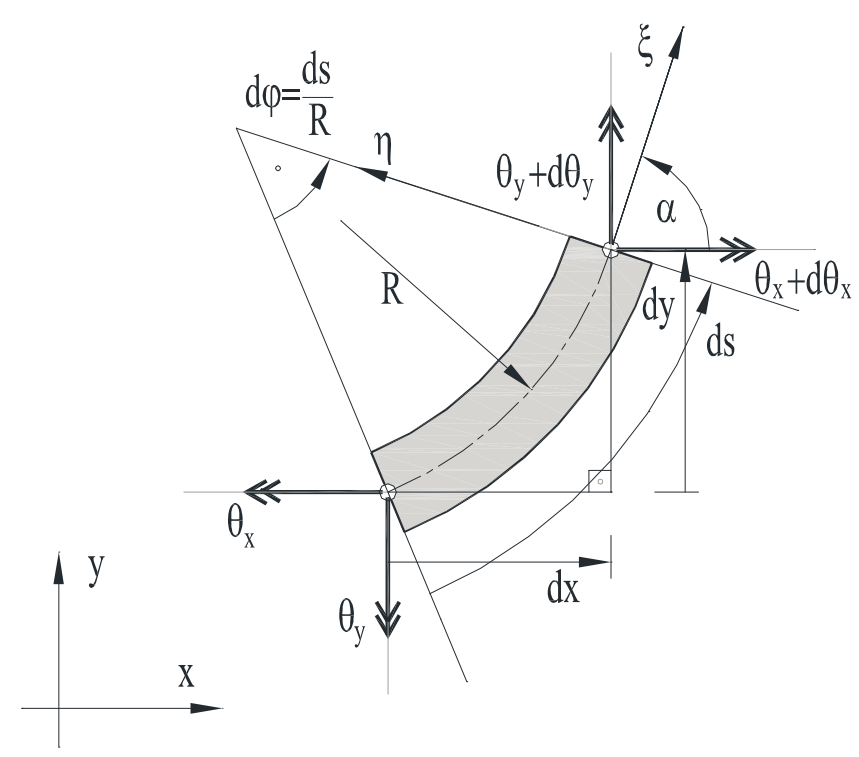

Fig. 5 Kinematics 2

Based on Fig. 5 the same deformation curvatures can be expressed ("kinematics 2") for direction $\eta$ : $\quad \kappa_{\eta} \cdot \mathrm{d} s=\mathrm{d} \theta_{y} \cos \alpha-\mathrm{d} \theta_{x} \sin \alpha$, and for direction $\xi: \quad \kappa_{\xi} \cdot \mathrm{d} s=\mathrm{d} \theta_{x} \cos \alpha+\mathrm{d} \theta_{y} \sin \alpha$. 


$$
\begin{aligned}
& \text { From } \cos \alpha=\frac{\mathrm{d} x}{\mathrm{~d} s} \text { and } \sin \alpha=\frac{\mathrm{d} y}{\mathrm{~d} s} \text { it follows: } \\
& \qquad \begin{array}{l}
\kappa_{\eta}=\frac{\mathrm{d} \theta_{y}}{\mathrm{~d} s} \frac{\mathrm{d} x}{\mathrm{~d} s}-\frac{\mathrm{d} \theta_{x}}{\mathrm{~d} s} \frac{\mathrm{d} y}{\mathrm{~d} s}=\left(\frac{\mathrm{d} \theta_{y}}{\mathrm{~d} \xi} \frac{\mathrm{d} x}{\mathrm{~d} \xi}-\frac{\mathrm{d} \theta_{x}}{\mathrm{~d} \xi} \frac{\mathrm{d} y}{\mathrm{~d} \xi}\right) \frac{1}{J^{2}}, \\
\kappa_{\xi}=\frac{\mathrm{d} \theta_{x}}{\mathrm{~d} s} \frac{\mathrm{d} x}{\mathrm{~d} s}+\frac{\mathrm{d} \theta_{y}}{\mathrm{~d} s} \frac{\mathrm{d} y}{\mathrm{~d} s}=\left(\frac{\mathrm{d} \theta_{x}}{\mathrm{~d} \xi} \frac{\mathrm{d} x}{\mathrm{~d} \xi}+\frac{\mathrm{d} \theta_{y}}{\mathrm{~d} \xi} \frac{\mathrm{d} y}{\mathrm{~d} \xi}\right) \frac{1}{J^{2}} .
\end{array}
\end{aligned}
$$

Here, $\alpha(\xi)$ is the angle between the $x$-global axis and the tangent at the curved beam point and is determined by local variable position $\xi$. Now, curvatures $\kappa_{\xi} \cdot \mathrm{d} s$ and $\kappa_{\eta} \cdot \mathrm{d} s$ can be expressed only by the differential changes of the global rotations and by differential beam geometry changes (5a) and (6a). The same result can be achieved directly from (5) and (6) after substituting the local section rotation with the global rotations and after introducing (13) and (17):

$$
\begin{aligned}
\cos \alpha(\xi) & =\frac{\frac{\mathrm{d} x}{\mathrm{~d} \xi}}{\frac{\mathrm{d} s}{\mathrm{~d} \xi}}=\frac{\frac{\Delta x}{2}+\Delta^{2} x \cdot \xi}{J}, \\
\cos \alpha(\xi) & =\frac{\frac{\mathrm{d} x}{\mathrm{~d} \xi}}{\frac{\mathrm{d} s}{\mathrm{~d} \xi}}=\frac{\frac{\Delta x}{2}+\Delta^{2} x \cdot \xi}{J} .
\end{aligned}
$$

Kinematics 2, formulated by (5a) and (6a), brings some obvious advantages over kinematics 1. It gives a possibility to interpolate the global rotations instead of the local rotations, thus eliminating the need for coordinate transformations of the element stiffness matrix from the local to the global coordinates, speeding up the calculation procedure. The resulting items of the stiffness matrix would have global orientations. Secondly, the interpolations for both rotations are treated equally as derivatives in (5a) and (6a) and would retain the same polynomial order, which is not the case with (5) and (6). The beam geometry interpolation is treated in the same way leaving a possibility of interpolating standard polynomials for the geometry of the curved beam and they can be defined just by nodal coordinates. There is no need to introduce any beam geometry curvatures or the curvature radius from (13) in further formulations.

\section{Linked interpolation for a three-node curved beam element}

Now, the linked interpolation procedure can be formulated as in [13] and [14] for a three-node beam element since only the elements with more than two nodes can model a curved form geometry. The transverse displacement is interpolated with a higher order polynomial than both rotations, since the cross-section bending rotation should equal the derivative of the displacement expression in the thin limit conditions (shear strain should vanish in (4)). Since the curved beam geometry is also involved, this interpolation should be cubic multiplied by the linear part ( $4^{\text {th }}$ order polynomial in local position variable $\left.\xi\right)$, as the Jacobian (14) coordinate transformation is also formulated with the linear components (as it was done in [15]).

$$
\begin{aligned}
& w=-\xi \frac{1-\xi}{2} w_{1}+\left(1-\xi^{2}\right) w_{2}+\xi \frac{1+\xi}{2} w_{3}+\frac{\xi-\xi^{3}}{4}\left(w_{b 1}+\xi \cdot w_{b 2}\right)= \\
& =w_{2}+\frac{\Delta w}{2} \xi+\frac{\Delta^{2} w}{2} \xi^{2}+\frac{\xi-\xi^{3}}{4}\left(w_{b 1}+\xi \cdot w_{b 2}\right),
\end{aligned}
$$


where linear and quadratic differences of displacements are $\Delta w=w_{3}-w_{1}$, $\Delta^{2} w=w_{1}-2 w_{2}+w_{3}$.

At the beginning, higher order terms (the cubic and the quartic in (18)) are related to some unknown internal parameters (degrees of freedom), signed with $w_{\mathrm{b} 1}$ and $w_{\mathrm{b} 2}$. The accompanying interpolations for global rotation components will be the standard Lagrangean functions passing through the three-node values. Nevertheless, opposite to other research approaches and with the benefit given by expressions (5a) and (6a), the interpolations will be set for global projections of the cross-sectional rotations as follows:

$$
\begin{aligned}
& \theta_{x}=\sum_{i=1}^{9} N_{i} \theta_{x i}=\theta_{x 2}+\frac{\Delta \theta_{x}}{2} \xi+\frac{\Delta^{2} \theta_{x}}{2} \xi^{2}, \\
& \theta_{y}=\sum_{i=1}^{9} N_{i} \theta_{y i}=\theta_{y 2}+\frac{\Delta \theta_{y}}{2} \xi+\frac{\Delta^{2} \theta_{y}}{2} \xi^{2},
\end{aligned}
$$

where $N_{i}$ are the standard Lagrangean quadratic interpolation functions associated with nodal rotation parameters and on the right there is its polynomial counterpart form and

$$
\Delta \theta_{x}=\theta_{x 3}-\theta_{x 1}, \Delta^{2} \theta_{x}=\theta_{x 1}-2 \theta_{x 2}+\theta_{x 3} \text { and } \Delta \theta_{y}=\theta_{y 3}-\theta_{y 1}, \Delta^{2} \theta_{y}=\theta_{y 1}-2 \theta_{y 2}+\theta_{y 3} \text {. }
$$

For a three-node curved beam element under out-of-plane actions the interpolations involve complete forth-order expansion in the beam transverse displacement $\mathrm{w}$ and quadratic in the rotations, using nodal parameters $w_{1}, w_{2}, w_{3}, \theta_{x 1}, \theta_{x 2}, \theta_{x 3}, \theta_{y 1}, \theta_{y 2}, \theta_{y 3}$ and two extra internal parameters $w_{b 1}$ and $w_{b 2}$, in total 11 independent degrees of freedom.

Shear strain can now be expressed from kinematic equation (4) and the global rotations are introduced instead of the local $\theta_{\eta}$ as

$$
\gamma_{z}=\frac{\mathrm{d} w}{\mathrm{~d} \xi} \frac{\mathrm{d} \xi}{\mathrm{d} s}-\theta_{x} \frac{\mathrm{d} y}{\mathrm{~d} \xi} \frac{\mathrm{d} \xi}{\mathrm{d} s}+\theta_{y} \frac{\mathrm{d} x}{\mathrm{~d} \xi} \frac{\mathrm{d} \xi}{\mathrm{d} s}=\left(\frac{\mathrm{d} w}{\mathrm{~d} \xi}-\theta_{x} \frac{\mathrm{d} y}{\mathrm{~d} \xi}+\theta_{y} \frac{\mathrm{d} x}{\mathrm{~d} \xi}\right) \frac{1}{J} .
$$

Introducing (18), (19) and (20) into (21a) a rational quartic polynomial expressing shear can be derived as follows:

$$
\begin{aligned}
& \gamma_{z}=\left\{\frac{\Delta w}{2}+\frac{w_{b 1}}{4}-\theta_{y 2} \frac{\Delta x}{2}+\theta_{x 2} \frac{\Delta y}{2}+\right. \\
& +\xi\left(\Delta^{2} w+\frac{w_{b 2}}{2}+\frac{\Delta \theta_{y}}{2} \frac{\Delta x}{2}+\theta_{y 2} \cdot \Delta^{2} x-\frac{\Delta \theta_{x}}{2} \frac{\Delta y}{2}-\theta_{x 2} \cdot \Delta^{2} y\right)+ \\
& +\xi^{2}\left[-\frac{3 w_{b 1}}{4}+\frac{\Delta^{2} \theta_{y}}{2} \frac{\Delta x}{2}+\frac{\Delta \theta_{y}}{2} \Delta^{2} x-\frac{\Delta^{2} \theta_{x}}{2} \frac{\Delta y}{2}-\frac{\Delta \theta_{x}}{2} \Delta^{2} y\right] \\
& \left.+\xi^{3}\left[-w_{b 2}+\Delta^{2} \theta_{y} \frac{\Delta x}{2}-\Delta \theta_{x} \frac{\Delta y}{2}\right]\right\} \frac{1}{J} .
\end{aligned}
$$

In order to eliminate the higher order terms associated with $\xi^{2}$ and $\xi^{3}$, which prevents the shear strain to take the linear form as it was done in [15], our free parameters $w_{b 1}$ and $w_{b 2}$ are now set to

$$
\begin{aligned}
& w_{b 1}=\frac{2}{3}\left(\Delta^{2} \theta_{y} \frac{\Delta x}{2}+\Delta \theta_{y} \Delta^{2} x-\Delta^{2} \theta_{x} \frac{\Delta y}{2}-\Delta \theta_{x} \Delta^{2} y\right), \\
& w_{b 2}=\Delta^{2} \theta_{y} \frac{\Delta^{2} x}{2}-\Delta^{2} \theta_{x} \frac{\Delta^{2} y}{2} .
\end{aligned}
$$


The beam shear strain gets now its final form:

$$
\begin{aligned}
& \gamma_{z}=\left\{\frac{\Delta w}{2}+\frac{1}{3}\left(\frac{\theta_{y 1}+4 \theta_{y 2}+\theta_{y 3}}{2} \frac{x_{3}-x_{1}}{2}-\frac{\theta_{x 1}+4 \theta_{x 2}+\theta_{x 3}}{2} \frac{y_{3}-y_{1}}{2}+\Delta \theta_{y} \frac{\Delta^{2} x}{2}-\Delta \theta_{x} \frac{\Delta^{2} y}{2}\right)+\right. \\
& +\xi \cdot\left(w_{1}-2 w_{2}+w_{3}+\frac{\theta_{y 3}-\theta_{y 1}}{2} \frac{x_{3}-x_{1}}{2}-\frac{\theta_{x 3}-\theta_{x 1}}{2} \frac{y_{3}-y_{1}}{2}+\right. \\
& \left.\left.+\frac{\theta_{y 1}+2 \theta_{y 2}+\theta_{y 3}}{2} \frac{\Delta x}{2}-\frac{\theta_{x 1}+2 \theta_{x 2}+\theta_{x 3}}{2} \frac{\Delta y}{2}\right)\right\} \frac{1}{J}
\end{aligned}
$$

The bending cross-section curvatures are easily derived from (5a) and (6a) as:

$$
\begin{aligned}
& \kappa_{\eta}=\left[\left(\frac{\theta_{y 3}-\theta_{y 1}}{2}+\Delta \theta_{y} \cdot \xi\right) \cdot\left(\frac{x_{3}-x_{1}}{2}+\Delta x \cdot \xi\right)-\right. \\
& \left.-\left(\frac{\theta_{x 3}-\theta_{x 1}}{2}+\Delta \theta_{x} \cdot \xi\right) \cdot\left(\frac{y_{3}-y_{1}}{2}+\Delta y \cdot \xi\right)\right] \frac{1}{J^{2}}, \\
& \kappa_{\xi}=\left[\left(\frac{\theta_{y 3}-\theta_{y 1}}{2}+\Delta \theta_{y} \cdot \xi\right) \cdot\left(\frac{y_{3}-y_{1}}{2}+\Delta y \cdot \xi\right)+\right. \\
& \left.+\left(\frac{\theta_{x 3}-\theta_{x 1}}{2}+\Delta \theta_{x} \cdot \xi\right) \cdot\left(\frac{x_{3}-x_{1}}{2}+\Delta x \cdot \xi\right)\right] \frac{1}{J^{2}} .
\end{aligned}
$$

Now the element stiffness matrix can be simply formed by the standard integration rule over the element domain with the B-type bar matrices from (24), (25) and (26):

$$
\begin{gathered}
\boldsymbol{\kappa}_{\eta}=\mathbf{B}_{B} \mathbf{u}_{\mathbf{n}}, \\
\boldsymbol{\kappa}_{\xi}=\mathbf{B}_{T} \mathbf{u}_{\mathbf{n}}, \\
\gamma_{z}=\mathbf{B}_{\gamma} \mathbf{u}_{\mathbf{n}},
\end{gathered}
$$

with vector $\mathbf{u}_{\mathbf{n}}$ as a vector of the unknown nodal degrees of freedom:

$$
\mathbf{u}_{\mathbf{n}}=\left\{w_{1}, \theta_{x 1}, \theta_{y 1}, w_{2}, \theta_{x 2}, \theta_{y 2}, w_{3}, \theta_{x 3}, \theta_{y 3}\right\}^{T} \text {. }
$$

The element stiffness matrix is then formed with three independent contributions, the bending energy part, the torsional energy part and the shear energy part:

$$
\mathbf{K}_{\mathbf{e}}=\mathbf{K}_{B}+\mathbf{K}_{T}+\mathbf{K}_{\gamma}=\int_{L e} \mathbf{B}_{B}^{T} E I_{B} \mathbf{B}_{\mathbf{B}} \mathrm{d} s+\int_{L e} \mathbf{B}_{T}^{T} G I_{T} \mathbf{B}_{T} \mathrm{~d} s+\int_{L e} \mathbf{B}_{\gamma}^{T} G k A \mathbf{B}_{\gamma} \mathrm{d} s
$$

The solution to some curved beam finite element model can now be reached by the standard minimization process of the total potential energy of the FEM model. Nevertheless, any plane beam problem can be structured with a chain of curved beam elements that can vary for a given curved geometry, determined only by its three nodal positions.

\section{Examples}

Hereinafter, the presented element will be named CBLI-3 after a curved beam element with linked interpolations generated for three nodes and will be further tested on some benchmark out-of-plane beam problems and compared whenever it is possible to the results of other curved beam elements from the literature. 
6.1 Quarter curved cantilever beam subjected to out-of-plane moment

Firstly, the present parabolic element $C B L I-3$ is tested on a simple numerical problem of a quarter of the circle clamped beam, subjected to tip loads on the beam free end. If moment load $M_{A, y}$ around the y-axis is applied with a bending effect on the cross-section in $A$ (Fig. 6), there is no shear strain involved in this problem, leaving bending and torsional strains to couple along the circular geometry of the beam.

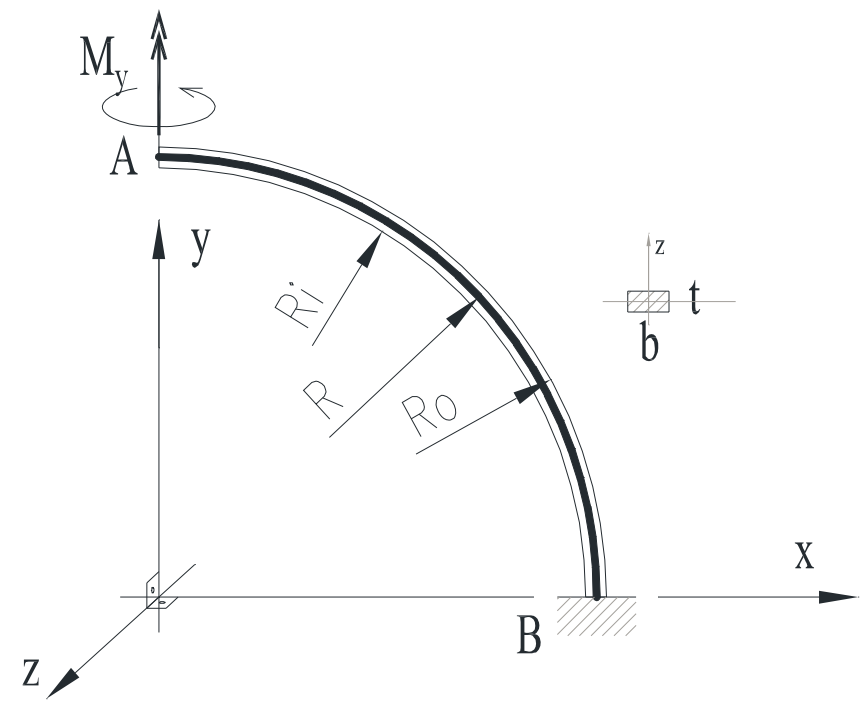

Fig. 6 A curved cantilever beam subjected to out-of-plane tip moment

The numerical data for this problem employed by MacNeal and Harder [16] are as follows: the inner radius is $R_{i}=4.12$, the outer radius is $R_{o}=4.32$, the thickness of the beam is $t=0.1$, the material data are $E=10 \mathrm{e} 7$, and $v=0.25$. A unit moment is applied at the free end. In this example, the cross-section of the beam is rectangular and its width can be evaluated by the radius difference, $b=0.20$.

The results of the finite element modelling involving one to up to eight proposed curved beam elements with a constant problem curvature are compared to the exact values for the tip node displacement and the two rotations about the global coordinate axes (correspond to the section bending and torsional rotations in $A$ ) that can be evaluated analytically from (32) to (34) and are given in Table 1 with the problem numerical data.

$$
\begin{aligned}
& w_{A}=\frac{M_{A, y} R^{2}}{2}\left(\frac{1}{E I_{B}}+\frac{1}{G I_{T}}\right), \\
& \theta_{y, A}=\frac{M_{A, y} R \pi}{4}\left(\frac{1}{E I_{B}}+\frac{1}{G I_{T}}\right), \\
& \theta_{x, A}=\frac{M_{A, y} R}{2}\left(-\frac{1}{E I_{B}}+\frac{1}{G I_{T}}\right) .
\end{aligned}
$$

Since $G=\frac{E}{2(1+v)}=0.4 \cdot E$ and $I_{T}=0.2289 \cdot b t^{3}=0.45776 \cdot 10^{-4}$, the torsional section stiffness is $G I_{T}=183.104$. At the same time the bending section stiffness is $E I_{B}=166.667$, so 
that actual stiffness ratio is $\frac{E I_{B}}{G I_{T}}=0.91$, giving both bending and torsional strains, almost equally contributing to the control values for $w_{A}$ and $\theta_{y A}$.

Table 1a Tip displacement and rotations for a quarter of the circle curved beam model with constant $R$ and tip moment $M_{y, A}=1$.

\begin{tabular}{|c|c|c|l|l|l|c|}
\hline \multirow{2}{*}{$\begin{array}{c}\text { Number of } \\
\text { nodes }\end{array}$} & \multicolumn{3}{|c|}{ CBLI-3 } & \multicolumn{3}{|c|}{ FRAME from FEAP [17] } \\
\cline { 2 - 7 } & $w_{A}$ & $\Theta_{x, A}$ & $\Theta_{y, A}$ & \multicolumn{1}{|c|}{$w_{A}$} & \multicolumn{1}{|c|}{$\Theta_{x, A}$} & $\Theta_{y, A}$ \\
\hline 3 & 0.1014088 & 0.0012355 & 0.0374932 & 0.095046 & 0.0012301 & 0.037019 \\
5 & 0.1020014 & 0.0011422 & 0.0379529 & 0.10028 & 0.0011588 & 0.037744 \\
9 & 0.1020509 & 0.0011368 & 0.0379852 & 0.10161 & 0.0011420 & 0.037926 \\
17 & 0.1020542 & 0.0011365 & 0.0379872 & 0.10194 & 0.0011379 & 0.037972 \\
\hline $\begin{array}{c}\text { Reference } \\
(32)-(34)\end{array}$ & 0.1020544 & 0.0011365 & 0.0379874 & 0.1020544 & 0.0011365 & 0.0379874 \\
\hline
\end{tabular}

Although the geometry of the quarter of the circle is modelled by parabolic element segments with CBLI-3, the convergence of the control data is rather fast even with the coarse element meshes, especially when compared with the typical two node constant strain beam element, as the one used in FEAP program [17] (see Table 1a) .

It has been reported in [8] that the curved elements can react with locking behavior if the section stiffness ratio reaches both extremes. Here we want to test this influence with the present curved beam element. This has been done by appropriate numerical choice of $I_{B}$ and $I_{T}$. The results of various flexure-to-torsion stiffness ratios are given in Table $1 \mathrm{~b} . w_{A h}$ is a finite element result for the model with 8 curved elements, and $w_{A}$ is the analytical solution from (32).

Table 1b Effect of the flexure-to-torsion stiffness ratio (EI/GJ) on the normalized transverse tip deflection of a curved cantilever beam subjected to out-of-plane unit tip moment, $M_{y, A}=1$.

\begin{tabular}{|c|c|c|c|c|c|c|c|c|c|}
\hline$E_{I B} / G_{I T}$ & $10^{-4}$ & $10^{-3}$ & $10^{-2}$ & $10^{-1}$ & 1 & $10^{1}$ & $10^{2}$ & $10^{3}$ & $10^{4}$ \\
\hline$w_{A h} / w_{A}$ & 0.9969 & 0.9977 & 0.9993 & 0.9999 & 1.0000 & 1.0001 & 1.0006 & 1.0023 & 1.0031 \\
\hline
\end{tabular}

These extreme ratios cannot be reached for an ordinary thick or thin beam section. For example, if the thickness of the section of the initial problem is taken $t=0.01$, so that $I_{T}=0.333 \cdot b t^{3}=0.6666 \cdot 10^{-7}$ and $G I_{T}=0.2667$, the corresponding bending section stiffness is $E I_{B}=0.1667$ and the resulting stiffness ratio is just $\frac{E I_{B}}{G I_{T}}=0.625$. Extreme ratios, for example $\frac{E I_{B}}{G I_{T}}=0.001$ or less, are unnatural as they are in the opposite ratio. Nevertheless, the presented element is obviously insensitive to the curvature-related locking on the flexure-totorsion stiffness ratios.

\subsection{Quarter curved cantilever beam subjected to out-of-plane tip shear load}

The next test problem will involve a shear strain part of the stiffness matrix. The same geometry is used for the curved beam model of the quarter of the circle beam as in the previous example, but this time loaded with the tip out-of-plane force at the free end of the beam (Fig. 7). 


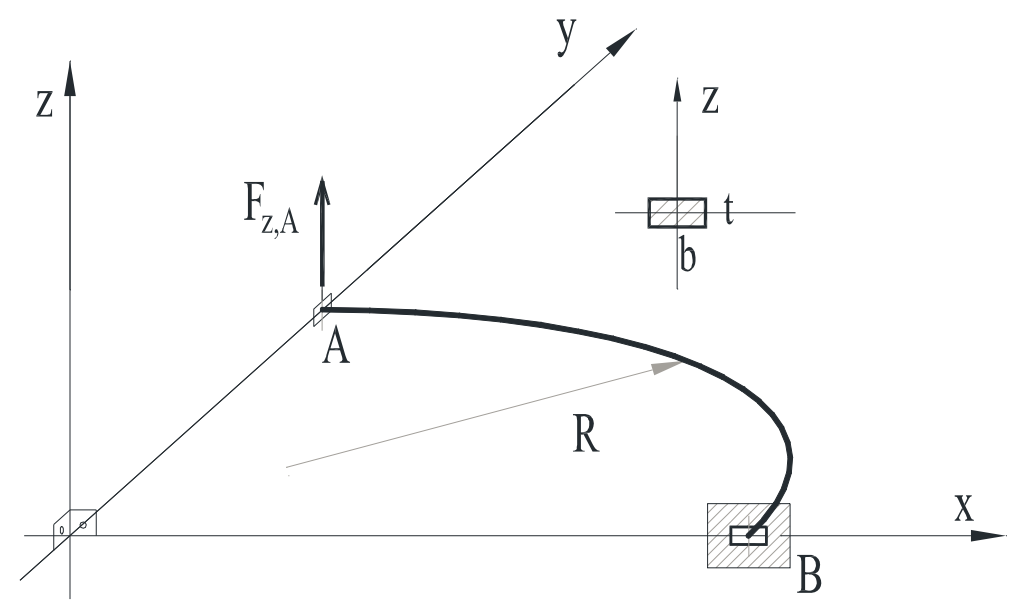

Fig. 7 A curved cantilever beam subjected to out-of-plane tip shear load

The force is of the unit magnitude and the analytical results are expressed as in [12]. This time shear strains are included in the analysis together with the bending and torsional parts, and the displacement and rotations at the beam free end are expressed as follows:

$$
\begin{aligned}
& w_{A}=-\frac{\pi}{2} \frac{F_{z} R}{G k A}-\frac{\pi}{4} \frac{F_{z} R^{3}}{E I_{B}}+\frac{F_{z} R^{3}}{G I_{T}}\left(2-\frac{3 \pi}{4}\right), \\
& \theta_{y, A}=-\frac{1}{2} \frac{F_{z} R^{2}}{E I_{B}}-\frac{1}{2} \frac{F_{z} R^{2}}{G I_{T}}, \\
& \theta_{x, A}=\frac{\pi}{4} \frac{F_{z} R^{2}}{E I_{B}}-\frac{F_{z} R^{2}}{G I_{T}}\left(1-\frac{\pi}{4}\right) .
\end{aligned}
$$

Moreover, the finite element model involves one to up to eight proposed curved beam elements and the results are shown in Table $2 \mathrm{a}$.

Furthermore, the convergence of the results is very fast despite the parabolic geometry interpolation of the circular beam. Additionally, the influence of the number of the Gauss integration points on the result convergences is analysed. The stiffness matrix is calculated from strain expressions (24) to (26) and the rational functions have to be integrated in (31). The nominators of the integrands are quartic functions in the bending and torsional parts, and are quadratic for the shear contribution. It can be concluded from Table $2 \mathrm{a}$ that three integration points suffice for the best integration calculus. Even with the two-integration point procedure, surprisingly good results are obtained. Actually, the results with two integration points are identical to the results of the 3-node curved beam element from the commercial FEM program (e.g. ANSYS). 
Table 2a Tip displacement and rotations for a quarter of the circle curved beam model with constant $R$ and tip moment $M_{y, A}=1$.

\begin{tabular}{|c|c|c|c|c|}
\hline $\begin{array}{c}\text { Number of } \\
\text { integration } \\
\text { points }\end{array}$ & $\begin{array}{l}\text { Number of CBLI- } \\
3 \text { elements }\end{array}$ & $w_{A}$ & $\Theta_{x, A}$ & $\Theta_{y, A}$ \\
\hline \multirow{4}{*}{2} & 1 & 0.4845123 & 0.0626041 & 0.1021005 \\
\hline & 2 & 0.4993775 & 0.0630208 & 0.1020576 \\
\hline & 4 & 0.5003683 & 0.0630466 & 0.1020546 \\
\hline & 8 & 0.5004313 & 0.0630482 & 0.1020544 \\
\hline \multirow{4}{*}{3} & 1 & 0.4890836 & 0.0620261 & 0.1014088 \\
\hline & 2 & 0.4997155 & 0.0629798 & 0.1020014 \\
\hline & 4 & 0.5003904 & 0.0630439 & 0.1020509 \\
\hline & 8 & 0.5004327 & 0.0630480 & 0.1020542 \\
\hline \multirow{4}{*}{4} & 1 & 0.4886466 & 0.0620956 & 0.1014542 \\
\hline & 2 & 0.4997071 & 0.0629812 & 0.1020027 \\
\hline & 4 & 0.5003902 & 0.0630440 & 0.1020509 \\
\hline & 8 & 0.5004327 & 0.0630480 & 0.1020542 \\
\hline \multirow{4}{*}{5} & 1 & 0.4886897 & 0.0620872 & 0.1014523 \\
\hline & 2 & 0.4997073 & 0.0629811 & 0.1020027 \\
\hline & 4 & 0.5003902 & 0.0630440 & 0.1020509 \\
\hline & 8 & 0.5004327 & 0.0630480 & 0.1020542 \\
\hline \multicolumn{2}{|c|}{ Reference (35)-(37) } & 0.5004353 & 0.0630483 & 0.1020544 \\
\hline
\end{tabular}

Next, on this test example, the existence of shear locking phenomena can be tested as in [8] and [3]. For this purpose, shear section stiffness $G k A$ is numerically changed from very large to very small, compared to bending section stiffness $E I_{B}$. Again, the influence of their ratio is tested for the normalized tip deflection at the beam free end and the results are presented in Table $2 \mathrm{~b} . w_{A h}$ is again a tip deflection of a finite element model with eight curved elements implied, and $w_{A}$ is the analytical solution from (35).

Table 2b Effect of flexure-to-shear stiffness ratio $\left(E I_{B} / G k A\right)$ on the normalized transverse tip displacement of a curved cantilever beam subjected to out-of-plane unit tip force, $F_{z}=1$ ( 8 elements used).

\begin{tabular}{|c|c|c|c|c|c|c|c|c|c|}
\hline$E I_{B} / G k A$ & $10^{-4}$ & $10^{-3}$ & $10^{-2}$ & $10^{-1}$ & 1 & $10^{1}$ & $10^{2}$ & $10^{3}$ & $10^{4}$ \\
\hline$w_{A h} / w_{A}$ & 1.0001 & 1.0000 & 1.0000 & 1.0000 & 1.0000 & 1.0000 & 1.0000 & 1.0000 & 1.0000 \\
\hline
\end{tabular}

Obviously, no shear locking is observed, not even for the extreme and unnatural ratios $E I_{B} / G k A$.

\subsection{Semi-circular beam fixed at its ends}

A nonhomogeneous solution for the statically indeterminate curved beam model is tested next. A semicircular arch with both ends fixed (at $A$ and $B$ in Fig. 8) is subjected to a uniform load along the arch axis and perpendicular to its plane. The radius of the beam geometry is $R=10.0 \mathrm{~m}$. The intensity of the uniform loading is $q_{z}=1.0 \mathrm{kN} / \mathrm{m}$ and the crosssection rigidities are $E I_{B}=0.98 \mathrm{GNm}^{2}, G I_{T}=0.82 \mathrm{GNm}^{2}$, and $G k A=6.0 \mathrm{GN}$. The convergence 
of the deflection, bending moments, torque and shear are studied at the discrete sections of the curved beam and compared to the analytical solutions.

The reference deflection of the arch midpoint at $\mathrm{C}$ has the following analytical expression:

$$
w_{C}=\frac{\pi^{2}}{8} \frac{q_{z} R^{2}}{G k A}+\left(1-\frac{2}{\pi}\right) \frac{q_{z} R^{4}}{E I_{B}}+\left(1-\frac{2}{\pi}-\frac{\pi}{2}+\frac{\pi^{2}}{8}\right) \frac{q_{z} R^{4}}{G I_{T}},
$$

The midpoint bending moment and the reactions at the beam fixed end at B are also checked for numerical consistency and are calculated from the general analytic expressions for shear force, bending moment and torque along the beam's axis:

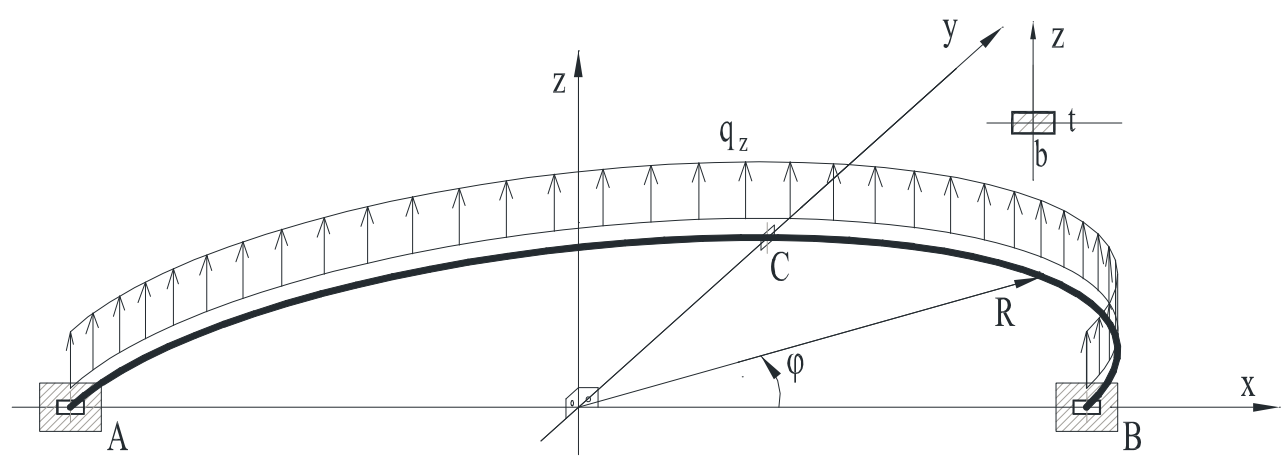

Fig. 8 A semi-circle cantilever beam subjected to out-of-plane uniform load

$$
\begin{aligned}
& V_{z}(\varphi)=q_{z} R\left(\frac{\pi}{2}-\varphi\right), \\
& M_{B}(\varphi)=-q_{z} R^{2}\left(1-\frac{4}{\pi} \sin \varphi\right), \\
& M_{T}(\varphi)=q_{z} R^{2}\left(\frac{\pi}{2}-\varphi-\frac{4}{\pi} \cos \varphi\right) .
\end{aligned}
$$

The stress resultants are defined by polar coordinate $\varphi$, measured from the clamped end as the origin. For the cross-sections at $B$ and $C$, the forces are calculated from the given geometric and material data. In Table $3 \mathrm{a}$, the finite element model results are tested for the presented element $C B L I-3$ and for other curved elements from literature dealing with the identical arch beam problem.

Then, the same problem is analysed in extremely flexible conditions, achieved for the model radii $R=100.0 \mathrm{~m}$. All other problem constants remain unchanged, and the behaviour of the present element is tested for the so-called thin limit condition.

Since $R_{b}=10 x R_{a}$ (see expressions (39)-(42)), the results in Table $3 \mathrm{~b}$ have $10^{4}$ times greater magnitude for $w_{C}, 10^{2}$ times greater for moments and 10 times greater for shear force. As the shear part of the displacement in (39) is only $10^{2}$ times greater, the result for $w_{C}$ is approaching the thin limit value for the Timoshenko beam theory, which is actually a Bernoulli's solution: $w_{C}=402.850 \mathrm{~m}$. At the same time, the results for stress resultants in Table $3 \mathrm{~b}$ are unaltered in precision for the same number of elements used. It must be also noticed that resultants in (40)-(42) are totally independent of beam material properties $E I_{B} G I_{T}$ and $G k A$. 
The CBLI-3 element shows no shear locking in the thin limit conditions, and is more precise in approximating beam displacement, while at the same time retaining a similar accuracy for controlled values of forces as the element of Sengupta-Dasgupta [1] or the element of Sheikh [7] or the element of Aköz-Kadioglu [5] for the problem with the nonhomogeneous solution with distributed load on the undetermined curved beam model.

Table 3a Convergence test of a semicircle beam fixed at its ends of $R=10.0 \mathrm{~m}$ and subjected to out-of-plane uniform load $q_{z}=1.0 \mathrm{kN} / \mathrm{m}$

\begin{tabular}{|c|c|c|c|c|c|c|}
\hline Element & $\begin{array}{l}\text { Number of } \\
\text { elements }\end{array}$ & $\begin{array}{l}w_{C} \\
\mathrm{~cm}\end{array}$ & $\begin{array}{l}M_{B, C} \\
\mathrm{kNm}\end{array}$ & $\begin{array}{l}M_{B, B} \\
\mathrm{kNm}\end{array}$ & $\begin{array}{l}M_{T, B} \\
\mathrm{kNm}\end{array}$ & $\begin{array}{l}V_{z, B} \\
\mathrm{kN}\end{array}$ \\
\hline \multirow{7}{*}{$\begin{array}{c}\text { Present } \\
\text { CBLI-3 } \\
\text { (with 3GP) }\end{array}$} & 1 & 3.83112 & 329.67 & -667.34 & 211.12 & 134.71 \\
\hline & 2 & 4.03407 & 300.20 & -890.64 & 298.14 & 149.97 \\
\hline & 4 & 4.04811 & 280.89 & -970.17 & 302.57 & 155.15 \\
\hline & 6 & 4.04888 & 276.72 & -986.52 & 300.63 & 156.21 \\
\hline & 8 & 4.04901 & 275.21 & -992.37 & 299.54 & 156.58 \\
\hline & 16 & 4.04906 & 273.74 & -998.08 & 298.15 & 156.95 \\
\hline & 20 & 4.04906 & 273.56 & -998.77 & 297.95 & 157.00 \\
\hline Dasgupta and & 6 & 4.04 & 301.34 & -993.07 & 265.63 & 157.07 \\
\hline Sengupta [1] & 20 & 4.04 & 275.85 & -999.81 & 294.89 & 157.07 \\
\hline $\begin{array}{c}\text { Akoz and } \\
\text { Kadioglu [5] }\end{array}$ & 20 & 4.09 & 223.73 & -1000.99 & 297.57 & 157.08 \\
\hline \multirow[t]{2}{*}{ Sheikh [7] } & 6 & 4.040 & 278.39 & -988.25 & 281.04 & 157.14 \\
\hline & 20 & 4.048 & 273.72 & -999.04 & 296.09 & 157.08 \\
\hline Analytical & $(39)-(42)$ & 4.04906 & 273.24 & -1000.00 & 297.56 & 157.08 \\
\hline
\end{tabular}

Table 3b Convergence test of a semicircle beam fixed at its ends of $R=100.0 \mathrm{~m}$ and subjected to out-of-plane uniform load $q_{z}=1.0 \mathrm{kN} / \mathrm{m}$

\begin{tabular}{|c|c|c|c|c|c|c|}
\hline Element & $\begin{array}{c}\text { Number of } \\
\text { elements }\end{array}$ & $\begin{array}{c}w_{C} \\
\mathrm{~m}\end{array}$ & $\begin{array}{c}M_{B, C} \\
\mathrm{kNm}\end{array}$ & $\begin{array}{c}M_{B, B} \\
\mathrm{kNm}\end{array}$ & $\begin{array}{c}M_{T, B} \\
\mathrm{kNm}\end{array}$ & $\begin{array}{c}V_{z, B} \\
\mathrm{kN}\end{array}$ \\
\hline Present & 1 & 381.113 & 32967. & -66734. & 21112. & 1347.1 \\
CBLI-3 & 2 & 401.374 & 30020. & -89064. & 29814. & 1499.7 \\
(with 3GP) & 4 & 402.776 & 28089. & -97017. & 30257. & 1551.5 \\
& 6 & 402.852 & 27672. & -98652. & 30063. & 1562.1 \\
& 8 & 402.865 & 27521. & -99237. & 29954. & 1565.8 \\
& 16 & 402.871 & 27374. & -99808. & 29815. & 1569.5 \\
& 20 & 402.871 & 27356. & -99877. & 29795. & 1570.0 \\
\hline Analytical & $(39)-(42)$ & 402.871 & 27324. & -100000. & 29756. & 1570.8 \\
\hline
\end{tabular}

\section{Conclusion}

A new three-node curved beam element CBLI-3 is presented for the analysis of nonstraight and straight linear plane structures under out-of-plane actions. The element is very simple and efficient in the FEM analysis, while at the same time very versatile in defining model geometry. It does not show any kind of extreme locking recognized in the literature, neither shear locking in the thin limit state, nor dominant bending or dominant torsion 
locking. The versatility of the geometry modelling is evident when, among other curved forms, the element can easily model a straight beam segment with the same stiffness matrix forming procedure. When modelling beam segments with a constant curvature radius (parts of the circle), the element with its parabolic geometry is not compatible with some other elements from the literature on coarse model meshes (one element mesh), but dense model meshes with two or four elements suffices for good results. On the other hand, higher order elements from the literature with a constant curvature are helpless when applied on the problems with variable geometry curvatures such as parabolic or upside catenary arches, which can alternatively easily be modelled with the presented element.

Alternative kinematic expressions are introduced relating the beam section strains to the global coordinate section rotations, which simplified all procedures in the element stiffness matrix formation.

The element model can easily be implemented in any programming code by the use of expressions (24)-(26) without reference to basic displacement interpolations and without intervention of reduced integration used by commercial programs, with no loss in accuracy in the strain and stress results.

\section{Acknowledgement}

The results of this study have been obtained within the research project IP-2016-064775: "Assumed strain method in finite elements for layered plates and shells within the application on layer delamination problem-ASDEL", financially supported by the Croatian Science Foundation, 2017.

\section{REFERENCES}

[1] S. Dasgupta and D. Sengupta, "Horizontally Curved Isoparametric Beam Element with or without Elastic Foundation Including Effect of Shear Deformation," Computers \& structures, vol. 29, no. 6, pp. 967-973, 1988. https://doi.org/10.1016/0045-7949(88)90322-7

[2] B. P. Naganarayana and G. Prathap, "Consistency aspects of out-of-plane bending, torsion and shear in a quadratic curved beam element," International Journal for Numerical Methods in Engineering, vol. 30, no. 3, pp. 431-443, 1990. https://doi.org/10.1002/nme.1620300304

[3] M. Ishaquddin, P. Raveendranath and J. N. Reddy, "Coupled polynomial field approach for elimination of flexure and torsion locking phenomena in the Timoshenko and Euler-Bernoulli curved beam elements," Finite Elements in Analysis and Design, vol. 65, pp. 17-31, 2013. https://doi.org/10.1016/j.finel.2012.10.005

[4] J. K. Choi and J. K. Lim, "General curved beam elements based on the assumed strain fields," Computers \& structures, vol. 55, no. 3, pp. 379-386, 1995. https://doi.org/10.1016/0045-7949(95)98865-n

[5] A. Y. Aköz and F. Kadioğlu, "The mixed finite element solution of circular beam on elastic foundation," Computers \& structures, vol. 60, no. 4, pp. 643-651, 1996. https://doi.org/10.1016/0045-7949(95)00418-1

[6] D. Sengupta and S. Dasgupta, "Static and dynamic applications of a five noded horizontally curved beam element with shear deformation," International Journal for numerical Methods in Engineering, vol. 40, pp. 1801-1819, 1997. https://doi.org/10.1002/(sici)1097-0207(19970530)40:10\%3C1801::aid-nme138\%3E3.0.co;2-v

[7] A. H. Sheikh, "New concept to include shear deformation in a curved beam element," Journal of Structural Engineering, vol. 128, no. 3, pp. 406-410, 2002. https://doi.org/10.1061/(asce)0733-9445(2002)128:3(406)

[8] M. Ishaquddin, P. Raveendranath and J. N. Reddy, "Flexure and torsion locking phenomena in out-ofplane deformation of Timoshenko curved beam element," Finite Elements in Analysis and Design, vol. 51, pp. 22-30, 2012. https://doi.org/10.1016/j.finel.2011.11.002

[9] T. Irie, G. Yamada and K. Tanaka., "Various Types of Out-of-plane Vibrations of Arcs," Bulletin of Faculty of Engineering, Hokkaido University, vol. 107, 1982.

[10] C. Huang, Y. Tseng, S. Chang and C. Hung, "Out-of-plane dynamic analysis of beams with arbitrary varying curvature and cross-section by dynamic stiffness matrix method," International Journal of Solids and Structures, vol. 37, pp. 495-513, 2000. https://doi.org/10.1016/s0020-7683(99)00017-7 
[11] B. Kim, C.-B. Kim, S. Song, H. Beom and C. Cho, "A finite thin circular beam element for out-of-plane vibration analysis of curved beams," J. of Mech. Science and Technology, vol. 23, pp. 1396-1405, 2009. https://doi.org/10.1007/s12206-008-1213-2

[12] E. Tufekci and O. Y. Dogruer, "Exact solution of out-of-plane problems of an arch with varying curvature and cross section," Journal of engineering mechanics, vol. 132, no. 6, pp. 600-609, 2006. https://doi.org/10.1061/(asce)0733-9399(2006)132:6(600)

[13] G. Jelenić and E. Papa, "Exact solution of 3D Timoshenko beam problem using linked interpolation of arbitrary order," Archive of Applied Mechanics, vol. 81, no. 2, pp. 171-183, 2011. https://doi.org/10.1007/s00419-009-0403-1

[14] E. Papa Dukić and G. Jelenić, "Exact solution of 3D Timoshenko beam problem: problem-dependent formulation," Archive of Applied Mechanics, vol. 84, no. 3, pp. 375-384, 2014. https://doi.org/10.1007/s00419-013-0805-y

[15] D. Ribarić and G. Jelenić, "Distortion-immune nine-node displacement-based quadrilateral thick plate finite elements that satisfy constant-bending patch test," International Journal for Numerical Methods in Engineering, vol. 98, no. 7, pp. 492-517, 2014. https://doi.org/10.1002/nme.4639

[16] R. H. MacNeal and R. L. Harder, "A proposed standard set of problems to test finite element accuracy," Finite elements in analysis and design, vol. 1, no. 1, pp. 3-20, 1985. https://doi.org/10.1016/0168-874x(85)90003-4

[17] O. Zienkiewicz and R. Taylor, "The Finite Element Method”, Fifth edition, Vulume 1: The Basis, Oxford: Butterworth-Heinemann, 2000. 